\title{
Organization in autobiographical memory
}

\author{
M. A. CONWAY and D. A. BEKERIAN \\ MRC Applied Psychology Unit, Cambridge, England
}

\begin{abstract}
Three experiments investigated timed autobiographical memory retrieval to cue words and phrases. In the first experiment, subjects retrieved memories to cues that named semantic category members and were primed with the superordinate category name or with a neutral word. No prime effects were observed. In the second experiment, subjects retrieved memories to primed and unprimed semantic category cues and to personal primes and personal history cues. Personal primes named lifetime periods (e.g., "school days") and personal history cues named general events occurring in those lifetime periods for each subject (e.g., "holiday in Italy"). Only personal primes were found to significantly facilitate memory retrieval. A third experiment replicated this finding and also failed to find any prime effects to primes and cues naming activities not directly related to an individual's personal history. In this experiment, characteristics of recalled events (e.g., personal importance, frequency of rehearsal, pleasantness, and specificity of the memory) were found to be strongly associated with memories retrieved to personal cues and only mildly associated with memories retrieved to other types of cues. These findings suggest that one way in which autobiographical memories may be organized is in terms of a hierarchically structured abstracted personal history.
\end{abstract}

Recent studies of autobiographical memory (Reiser, Black, \& Abelson, 1985; Robinson, 1976; Schank, 1982) have suggested ways in which autobiographical memories may be organized in memory. Schank emphasized the role of contexts and actions in the access and representation of specific autobiographical memories. Similarly, Robinson proposed that autobiographical memories may be organized into categories of events. The purpose of the present study was to examine these proposals in more detail and to consider related aspects of autobiographical memory organization. In particular, it was decided to investigate the role of an individual's personal history in the organization of specific autobiographical memories.

Schank (1982) proposed that autobiographical memories may be encoded and retrieved in terms of actions and goals involved in the original experience of an event. According to this view, autobiographical memories may be organized hierarchically, and different levels of the hierarchy detail increasingly more specific contexts terminating in autobiographical memories (see also Kolodner, 1983). Reiser et al. (1985) investigated the hierarchical organization of autobiographical memory by varying the specificity of contextual information used to cue autobiographical memory retrieval.

Reiser et al. (1985) distinguished between general actions and activities. General actions are characterized as sequences of actions that may be undertaken in a variety of contexts, for example, "finding a seat." Activities, on the other hand, refer to actions undertaken in specific contexts, for example, "going to the cinema." Reiser

This research was supported by the Medical Research Council of Great Britain. We would like to thank Endel Tulving, John Robinson, and two anonymous reviewers for helpful comments on earlier versions of this paper. Address correspondence to M. A. Conway, MRC Applied Psychology Unit, 15 Chaucer Road, Cambridge CB2 2EF, England. et al. found that when subjects were required to recall an autobiographical memory that matched a cue containing both an activity and a general action (e.g., "going to the cinema... finding a seat"), memory retrieval times were faster when presentation of the activity preceded presentation of the general action. Reiser et al. argued that the prior presentation of an activity cue facilitates retrieval by providing information that corresponds directly to the way in which the target memory is represented and by providing information that retrieval processes can effectively exploit in the search for a target memory. In contrast, general actions do not directly correspond to the representation of target memories, and information contained in the general actions must be elaborated in the course of retrieval. Thus, Reiser et al.'s findings tend to support the view that autobiographical memory may be hierarchically organized and that actions in context provide powerful cues for memory retrieval.

It is unclear, however, whether activity cues are the most effective means, or the sole means, by which autobiographical memories may be retrieved. Introspection suggests that short phrases or single words may act as powerful memory cues in everyday contexts. Robinson (1976) investigated autobiographical memory retrieval times cued by single words (cf. Galton, 1883) that named objects, activities, and feelings. One of Robinson's principal findings was that retrieval times to object and activity cues were significantly shorter than retrieval times to cues naming feelings. One explanation for this effect considered by Robinson relates to the hypothesized organization of autobiographical memory. If autobiographical memories are organized in categories of experiences indexed by a hierarchy of event categories, then one of the determinants of retrieval time might be the number of different memories associated with a particular cate- 
gory. Because more experiences, and hence more memories, involve feelings, the longer retrieval times to words naming feelings may be a product of a category size effect (Landauer \& Freedman, 1968).

This view implies that autobiographical memory retrieval may be mediated by some process of enumeration. For example, spreading activation models (Anderson, 1983; Collins \& Loftus, 1975) propose that activation diffuses as more nodes in the network are activated. This would lead to weaker activation of members of a large category than of members of a smaller category, and hence to retrieval time differences. Kolodner (1983, p. 247), however, argued that retrieval processes sensitive to some form of enumeration would not be expected to show the characteristic errors typically observed in autobiographical memory retrieval, for example, partial recall, "overshoots" (cf. Williams \& Hollan, 1981), and the successive generation of contextually related retrieval cues. Furthermore, Reiser et al. (1985) found that highly specific cues, such as "went to the cinema and did not find a seat," which presumably map onto very few autobiographical memories, produced very long retrieval times. According to the enumeration view, these cues, of course, should produce the shortest retrieval times. However, one problem with Reiser et al.'s (1985) manipulation is that actions that fail may not be well integrated with knowledge structures organizing autobiographical memories. Retrieval of these memories may require considerable elaboration before any memories are searched, hence the longer retrieval times. Thus, the possibility remains that enumeration may occur, but only for sets of memories that are well integrated with existing knowledge structures.

It is difficult to see how current models of autobiographical memory (e.g., Kolodner's [1983] directed search model; Reiser et al.'s [1985] context plus index model) can readily account for the lengthy retrieval times to emotion word cues. Thus, the proposal that autobiographical memories may be loosely organized into categories, such as Feelings, and that category size may determine retrieval time, remains a possibility. It is, of course, a well-established finding that other types of knowledge (e.g., semantic knowledge) may be represented in "fuzzy" categories (see Smith \& Medin, 1981, for a review). Given that at least some memorial information is represented in such knowledge structures, it does not seem unreasonable to suggest that autobiographical memories may be contained in the same knowledge structures, rather than represented in some form of contextually based knowledge structure. One purpose of the present study, then, was to examine whether autobiographical memories are conjointly represented with knowledge structures such as semantic categories.

Another possible form of autobiographical organization may be based upon general information that is specific to an individual. For instance, cues that contain information relating to an individual's personal history, whether or not those cues specify actions and contexts, may facili- tate memory retrieval. This suggestion implies that autobiographical memory may be organized according to some form of abstracted personal history, rather than in terms of actions and/or contexts per se. Hierarchical models that emphasize contexts and actions (e.g., that of Reiser et al., 1985) could, of course, accommodate such a form of organization; however, some modification of the models would be necessary to account for the organizing role of an individual's life context.

These aspects of autobiographical memory organization might be studied further in experiments involving priming of memory retrieval. For example, a prime such as "Feelings" may facilitate retrieval of a memory to the cue "happy" if autobiographical memories are organized in such categories. Similarly, prior presentation of a prime such as "going shopping" should facilitate memory retrieval to the cue "paying at a cash register" if autobiographical memory is hierarchically organized in terms of actions and contexts. A further possibility is that information that relates to an individual's personal history, such as "school days," may prime memory retrieval to cues that specify sets of experienced events, such as "math class," if autobiographical memories are organized in terms of personal history. In the present series of experiments, we investigated autobiographical memory organization by examining what types of primes facilitate the process of autobiographical memory retrieval.

\section{EXPERIMENT 1}

As an initial step, it was decided to conduct a simple word-word priming study. Subjects were required to retrieve an autobiographical memory to a cue word that named a category member; cue words were primed by the category name. The three categories employedSports, Furniture, and Emotions-were similar to the categories used by Robinson (1976). It was reasoned that if autobiographical memories were organized in such categories, then prior presentation of the category name would facilitate memory retrieval. For instance, prior presentation of the prime "Furniture" would facilitate autobiographical memory retrieval to the cue "chair," compared to retrieval after prior presentation of a neutral prime, such as "Ready."

The three categories used in the present study differed from those used by Robinson (1976), however, in that they were selected from sources that had established their status as semantic categories (Battig \& Montague, 1969; Conway \& Bekerian, in press; Fehr \& Russell, 1984; Hampton \& Gardiner, 1983; Rosch, 1975). One of our reasons for selecting these categories was that reliable category prime effects had been observed before, in tasks featuring semantic judgments, with these categories (Conway \& Bekerian, in press; Fehr, Russell, \& Ward, 1982; Rosch, 1975). Furthermore, the use of semantic categories allowed an additional manipulation: it was decided to vary the typicality of exemplars used to cue autobiographical memory retrieval. If autobiographical memories are or- 
ganized in semantic categories, then memories might be more closely associated with highly typical category exemplars. Thus, primed retrieval to typical category exemplars might be faster than primed retrieval to atypical category exemplars (e.g., Rosch, 1975). Such a finding would provide additional support for the proposal that autobiographical memories may be organized in terms of semantic categories.

\section{Method}

Subjects. Sixteen subjects, 14 females and 2 males, were recruited from the subject panel of the Applied Psychology Unit. All were between the ages of 20 and 39 and were paid for their participation in the experiment.

Design. A 2 (between-subjects) $\times 2 \times 3 \times 4$ (within-subjects) mixed design was employed. The first factor, which was a betweensubjects variable, was order: cues appearing in the prime condition in Order 1 appeared in the no-prime condition in Order 2, and vice versa. All other manipulations were within-subjects variables. In the prime condition, cues were preceded either by a prime denoting the category name or by a neutral word. Three categories of cues were used: Sports, Furniture, and Emotions. Level of typicality of the cue was also manipulated: half the cues were highly typical category members and half were atypical category members. Nested within categories and typicality was items, and this factor comprised the four cue words employed in each condition. The dependent variables were time taken to recall autobiographical memories and the age of memories.

Stimuli and Apparatus. The cues (exemplars) for Sports and Furniture were selected from Hampton and Gardiner (1983). The target items for Emotions were selected from Conway and Bekerian (in press). Sixteen items were selected for each category, eight highly typical and eight atypical. Common items that subjects would have encountered or experienced fairly frequently were chosen. (See Appendix for the full list of stimuli.) In each category, half the targets were randomly allocated to the prime condition. This allocation procedure was undertaken for each subject.

A separate group of exemplars, selected from categories other than those employed in the experiment, was used for demonstration and practice trials only. The experiment was run on an APple II microcomputer. Times were recorded in centiseconds.

Procedure. The experiment was conducted in a soundproofed cubicle. In the cubicle, a computer terminal was positioned on a desk so that the center of the screen was at eye level when the subject was seated directly in front of the terminal. Also on the desk was a single-key response box.

The subjects were told that the task was to recall a memory of a specific experience from the past in response to a single-word cue. In order to make this task easier, each word would be preceded by a warning signal. On some occasions the warning signal would be a meaningful word. On other occasions the warning signal would be the word "Ready." It was explained that in all cases the warning signal would be on-screen for a short time only, and that subjects should focus their attention on the center of the screen whenever a warning signal appeared. It was aiso explained that written beneath each cue word would be the instruction "Recall a personal experience."

The subjects were told that as soon as they had brought to mind a memory of a specific experience in response to the cue word they were to press the response key. They always pressed the response key with their dominant hand. It was explained that the computer was timing, in centiseconds, the time taken to recall an experience and that it was essential that they respond as soon as any memory came to mind.

As soon as the response key was pressed, the screen was blanked and the instruction "Make a brief note of your memory" appeared .
The subjects were instructed to write the cue word and one or two short sentences describing the recalled experience. All notes were written in a lined booklet provided by the experimenter. The subjects were instructed to make notes that, read later in the experiment, would remind them of the memories they had recalled. It was emphasized that during the course of the experiment only the subject would have access to his/her notes.

The subjects were also informed that if no memory came to mind within approximately $1 \mathrm{~min}$, they were to press the response key and write an $X$ in the booklet. It was emphasized that it was unlikely that subjects would be unable to recall memories.

After making a memory note, the subject pressed the response key for the next trial. A pretrial warning signal $(\mathrm{XXXX})$ then briefly appeared on the screen, followed by the next experimental trial.

This procedure was demonstrated in 12 practice trials, half of which were supervised by the experimenter. After every 12 trials subjects were given a 45 -sec rest. Including practice trials, there were five blocks of 12 trials.

The procedure for each trial was as follows: A pretrial warning signal was displayed for $150 \mathrm{csec}$; after a 150 -csec interval, during which the screen was blank, a prime was displayed for $150 \mathrm{csec}$; this was followed by an interstimulus interval of $75 \mathrm{csec}$. The target word was then displayed until the subject responded. The intertrial interval, during which the memory notes were made, was under the subject's control.

After the experimental trials, the subjects were asked to date to the nearest month the age of each memory. In order to do this the subjects consulted their memory notes. Finally, the subjects were asked if they wished to keep their memory notes. The whole experiment lasted approximately $1 \mathrm{~h}$.

\section{Results and Discussion}

The two dependent variables of interest were retrieval times of autobiographical memories and age of autobiographical memories. Factors to be analyzed were order, primes, categories, typicality, and items, with order a between-subjects variable. Items were nested within categories and typicality and were treated as a random factor. The $2 \%$ of missing cases, in which a subject had been unable to recall a memory, were replaced by that subject's mean in that condition. The distribution of retrieval times was examined prior to analysis.

Retrieval times. The overall distribution of retrieval times showed a positive skew of 36.05 with a very high kurtosis of 50.60 . The skew was a product of a very long tail to the distribution. This tail comprised 36 memory retrieval times, the shortest being $1,836 \mathrm{csec}$ and the longest being $4,778 \mathrm{csec} .{ }^{1}$ The five longest retrieval times were to different emotions. However, these excessively long retrieval times were not specific to any one subject or to any one item. It appeared that different subjects occasionally experienced difficulty in recalling a memory to different items. The kurtosis was a product of a large number of retrieval times (45\%) occurring in the range of $1-2 \mathrm{sec}$. Over $75 \%$ of retrieval times occurred in the range of $0.5-3 \mathrm{sec}$.

The only effect that proved to be significant was that of category $\left[\min F^{\prime}(2,45)=9.80, p<.01\right]$. Sports produced the fastest retrieval times $(229 \mathrm{csec})$, followed by Furniture $(282 \mathrm{csec})$ and Emotions (358 csec). Overall mean retrieval time was $290 \mathrm{csec}$. 
Mean retrieval times for prime and no-prime conditions were $302 \mathrm{csec}$ and $277 \mathrm{csec}$, respectively. Thus, a large but nonsignificant negative priming effect of $25 \mathrm{csec}$ was observed. Mean retrieval times to typical and atypical exemplars were $313 \mathrm{csec}$ and $266 \mathrm{csec}$, respectively, constituting an even larger nonsignificant negative priming effect of $47 \mathrm{csec}$.

Clearly nonsignificant differences approaching half a second indicate extreme variation in subjects' responses to the experimental manipulations. Inspection of the raw data showed that all subjects exhibited some large positive priming effects for some items. Some subjects showed a large percentage of positive priming effects, whereas others showed only a small percentage. Percentages varied across items and no single prime-target pair consistently produced positive priming effects. Mean retrieval times, across items, for each subject showed 9 subjects with negative priming effects, 4 subjects with positive priming effects, and 3 subjects who were equally fast on primed and unprimed trials. A similar pattern was evident for negative priming effects. Variation was observed for items of varying typicality, with 5 subjects markedly faster to retrieve memories to atypical exemplars. The remaining subjects showed small effects in both directions.

Dating and memory notes. In order to analyze the dating data, the age of each memory was standardized in the following way. The age of a memory in months (backdated from the time of recall) was divided by the total age of the subject (also in months) and the product was subtracted from 1 . Thus each memory age was expressed as a number between 0 and 1 , with a higher number indicating a more recent memory. This transformation had the advantage of expressing the age of a memory in terms of a proportion of the subject's life, thus making the ages of memories for subjects of differing ages more comparable. Missing ages of memories, which totaled less than $3 \%$, were replaced by the mean age for that subject in that condition. In the few cases in which subjects had dated a memory by season, the midpoint of the season was taken as being the date for that memory.

The distribution of the transformed ages was found to be negatively skewed, with a skew of 3.50 and a kurtosis of 5.56. A large recency effect in memory ages was observed: $50 \%$ of the memories were of events that had occurred within the preceding 12 months, $16 \%$ dated from the first half of the subjects' lives, and the remaining $34 \%$ were fairly evenly distributed over the period from the midpoint of the subjects' lives to 12 months prior to the experiment. This distribution of memory ages was similar across the three categories.

The standardized memory ages were entered into an analysis of variance identical to that employed for retrieval times, with $p<.02$ (as recommended by Keppel, 1973, p. 75). No significant effects were observed and memory ages across categories were very similar. Sports produced older memories than did the other two categories, with a mean age of 0.82 compared to mean ages of 0.88 for Furniture and 0.89 for Emotions.
An analysis of covariance was conducted in order to determine whether memory retrieval times correlated with age of memories. A significant effect of categories was present $[F(2,29)=17.7, p<.01]$, indicating that there was an effect of categories on autobiographical memory retrieval times over and above any effect of the age of memories. No other significant effects were found.

Fourteen of the 16 subjects' memory notes were available for inspection. The formal analysis of this data is reported elsewhere (Bekerian \& Conway, 1987a); however, some general comments about these notes are relevant here. First, many of the memory descriptions (about $60 \%$ ) appeared to refer to mundane, everyday events. The remainder of these descriptions referred to novel and unusual episodes that appeared to be marked by distinctive features. Memories of mundane and everyday events were more closely associated with positive than with negative priming effects, whereas memories of novel and unusual events were equally distributed across positive and negative priming effects.

Summary. Cued retrieval from autobiographical memory was not found to be affected by the prior presentation of a related semantic prime. Similarly, typicality of cue was not found to be related to retrieval time. The only significant effect was that of type of category cue, and retrieval times to words naming items in the category Emotions were significantly longer than retrieval times to words naming items of Furniture and Sports. This category effect replicates the finding of Robinson (1976).

The failure of a semantic prime to affect retrieval from autobiographical memory would seem to argue that autobiographical memories are not organized in, or accessed by way of, semantic categories. The significantly longer retrieval times to Emotion cues may indicate that memories of emotional experiences are typically accessed in some other way, for example, by instantiation of a mood state (Bower, 1981) or by more complex cues (Conway \& Bekerian, in press). Overall, large variances in retrieval times were observed, and differences approaching half a second failed to reach significance. Given such variance, it could be argued that in the lengthy and complex process of autobiographical memory retrieval, comparatively small priming effects either dissipate or are undetectable (cf. de Groot, 1983).

Clearly, many explanations for the variability in retrieval times could be considered. Given the failure to observe systematic and general priming effects upon autobiographical memory retrieval in Experiment 1, however, it seemed important to establish that autobiographical memory retrieval can in fact be primed. Experiment 2 attempts such a demonstration.

\section{EXPERIMENT 2}

Common experience suggests that retrieval of autobiographical memories can be primed, especially when the prime corresponds to a set of experienced events. For example, everyday conversations between people featur- 
ing topics such as "school days," "when at university," "holidays," and so on, frequently give rise to the recall of specific experiences. Indeed, Reiser, Black, and Kalamarides (1986), in a study in which subjects reported their thoughts while recalling memories, found that personal information relating to an individual's life history featured strongly in the recall process.

These types of memory cues, however, differ from those employed in Experiment 1 in a number of ways. In particular, the former cues contain information specifying contexts and time periods. Such cues are similar to those employed by Reiser et al. (1985) and discussed by Schank (1982), but differ in that they specify contexts, time periods, and actions. For example, the cue "school days" presumably encompasses context (specific schools), actions (typical school-related activities), and time period. The cue "school days," however, may refer not to any single specific memory, but rather to a number of experiences. It may be, then, that cues that refer to a set of experiences could be employed in such a way as to prime retrieval of related autobiographical memories.

The present study investigated this by employing primes that denoted common themes for sets or types of experiences. It was decided to collect from individual subjects names of lifetime periods and also lists of general events (personal history cues) that had occurred in various lifetime periods. (Pilot studies had suggested that subjects systematically distinguish between thematic periods in their lives, such as "when I worked at ___," and extended events that occurred during these periods, such as "eating at _'s on Fridays."') Note that Reiser et al. (1986) drew a similar distinction between time eras and larger contexts in accounting for their subjects' recall protocols. We predicted that prior presentation of a word or phrase naming a lifetime period would facilitate autobiographical memory retrieval to a related personal history cue, compared to the unprimed presentation of a personal history cue.

A further purpose of Experiment 2 was to extend the range of semantic primes and cues employed in Experiment 1. Although it seems unlikely, our failure to find priming effects in that experiment may have been specific to the three categories from which memory cues were drawn. In order to explore this possibility, we decided to include a larger sample of semantic category primes and exemplars (memory cues) in the present study.

\section{Method}

Subjects. Thirty-one subjects, all volunteer Cambridge undergraduates, were selected to take part in the experiment. The responses of 3 subjects to the personal memory questionnaire proved unsuitable, and 2 other subjects were unavailable for the experiment itself. The final subject sample was made up of 20 males and 6 females, giving a total of 26 subjects with a mean age of 21.7 years.

Design. After stimuli had been selected from the subjects' responses to the personal memory questionnaire (see below), the following priming experiment was constructed. A $2 \times 2 \times 10$ within-subjects design was employed. The first factor was the presence or absence of a prime. The two types of prime were lifetime periods and semantic category names. The word "ready" was used as the "prime" in the no-prime condition. The second factor referred to the type of cue following the prime. These were either personal history cues or semantic cues (highly typical semantic category members). The third factor was the number of items within each of the four subgroups: primed personal history cues, unprimed personal history cues, primed semantic cues, and unprimed semantic cues. There were 10 items (cues) in each group. The subjects were required to retrieve specific personal memories to each cue. The dependent variable was time taken (in milliseconds) to recall a memory.

Stimuli collection. In order to construct the stimuli for the priming experiment, subjects completed a two-part personal memory questionnaire. In the first part, the subjects were instructed to list 10 lifetime periods. They were told:

Most people, when thinking about or talking about their lives, tend to divide up their personal history into very general time periods, for example, "when I was at college." Obviously, such time periods are not mutually exclusive and frequently overlap each other. For instance, the period "when I played football" might overlap with "when I was at college." However, one characteristic of such periods is that they generally have a distinct beginning and end. That is to say, when we look back we can identify sets of personally experienced events which marked the start of, and close of, a general period. In this experiment we would like you to list 10 of these general periods from your life. Try to select the periods so that, collectively, they span your life.

The subjects then printed a different lifetime period at the head of each page of a 10-page booklet. They were instructed to try to name each lifetime period with a single word or short phrase. This part of the questionnaire took about $10 \mathrm{~min}$.

The subjects were then instructed to go back through the booklet and write down four or five general events (personal history cues) that happened to them during each lifetime period. They were told:

The events we would like you to list should refer to time periods longer than a couple of hours but not longer than one or two months. Typically these general events should refer to time periods lasting a day, a few days, or a week or so. You should not include very specific memories relating to short time periods such as a memory of a conversation.

This part of the questionnaire took about $30 \mathrm{~min}$ and all subjects found the questionnaire easy to complete.

Although no formal analysis of lifetime periods and personal history cues was undertaken, a few comments can be made about the nature of the subjects' responses. Collectively, the 10 lifetime periods listed by each subject covered all of the subject's life. The most commonly listed lifetime periods referred to preschool and school periods (e.g., "primary school," "secondary school," and "sixth form' "). All subjects named at least one of these periods and most named all of them. Other frequently named lifetime periods referred to locations in which the person had previously lived, to friends (in particular, past boy- and girlfriends), and to periods spent living outside Britain. Somewhat less frequently named was "my year off," referring to the practice of students to take a year out of education prior to going to university. However, all subjects named a number of idiosyncratic lifetime periods that were specific to individual subjects. Approximately $70 \%$ of all lifetime periods were named by all subjects.

Very little commonality was noted among the personal history cues listed by the subjects for their lifetime periods. The events listed had most often lasted for a day or longer, for example, "firstyear exams," "going to London," "trip to Scotland," "first week at primary school," "holiday in Italy." Although these events differed in their details from subject to subject, they were similar in type, naming short but distinctive periods involving journeys, being with friends, leisure activities, and new experiences.

Stimuli selection. Stimuli for the priming experiment were selected in the following way: Subjects who had failed to list 10 lifetime periods and corresponding personal history cues were dis- 
carded, as were subjects whose handwriting was illegible. The data from 3 subjects were lost because of these factors. For the remaining 28 subjects, individual sets of primes (lifetime periods) and cues (personal history cues) were created. In all cases, statements longer than two words naming a lifetime period were shortened to one or two words. For example, "when we lived in Italy" was shortened to "Italy," and "when I was in the sixth form" was shortened to "sixth form." About $50 \%$ of the lifetime period names were shortened in this way. Personal history cues, which were generally longer than two words, were reduced to a short phrase summarizing the event. For example, "when Jenny and I went on holiday" became "holiday with Jenny," and "playing football for the first team" became "first team football." Over 70\% of the personal history cues were shortened in this way. Personal history cues varied in length from one to six words.

For each subject, two personal history cues were randomly selected for each lifetime period from the three to five listed events. Each personal history cue was randomly allocated to the prime or no-prime condition. After this allocation the personal history cues in the two conditions for each lifetime period and for each subject were inspected. In cases where there was an imbalance in word length, that is, one condition contained all long personal history cues, the cues were reallocated so that each condition contained an equal number of short and long personal history cues. No individual subject's set of primed and unprimed personal history cues contained an extreme number of either short or long cues, although all subjects varied in the total number of short and long personal history cues included in their stimulus sets.

In this way a set of 20 primes plus cues was created for each subject. The primes were always lifetime periods or the word "ready" and the cues were always personal history cues. Ten of the cues were primed with their corresponding lifetime periods; the remaining 10, which were matched to the same lifetime periods, were paired with the word "ready" (no-prime condition).

The semantic prime and cue pairs were constructed in the following way: Ten categories were selected from Battig and Montague (1969) and Conway and Bekerian (in press). The categories were Flowers, Trees, Sports, Clothes, Fruits, Relatives, Vegetables, Vehicles, Furniture, and Emotions. For each category, 2 highly typical exemplars were randomly selected from the 10 most frequently named exemplars. For each exemplar, a short phrase (one to three words) and a long phrase (four to six words) were constructed. For example, for Furniture, the exemplar "desk" was contained in two phrases, "a desk" (short) and "sitting at a desk" (long). Long phrases generally named a common activity associated with the object.

For each subject 10 exemplars, one from each category, were randomly assigned to the prime condition and the remaining 10 to the no-prime condition. The short and long phrases containing the exemplars were then assigned to the two conditions in such a way as to ensure that each condition contained an equal number of short and long phrases. The number of short and long phrases in each condition approximated the number of short and long phrases contained in the corresponding conditions for lifetime periods and for personal history cues. Thus 20 prime-plus-cue pairs were created in which the prime was either a superordinate category name or the word "ready" and the cues were short or long phrases containing a typical exemplar of one of the 10 categories.

For each subject, then, a set of 40 primes and cues was constructed, half drawn from the personal memory questionnaire and half drawn from semantic categories.

Apparatus. The experiment was run on a BBC Acorn plus Torch Z80 microcomputer system using a programmable tachistoscope (Norris, 1984). A response box containing a single response key was also employed. The stimuli were displayed in white against a black background on a video display unit and all were in uppercase letters.
Procedure. The subjects were contacted 4 to 5 months after completing the personal memory questionnaire and were asked to take part in the experiment. Two of the 28 subjects were unavailable.

Each subject was seated at a table containing a monitor and a single-key response box, and was given written instructions that outlined the sequence of events on any one trial of the experiment. The sequence of events was as follows: A prime was displayed in the center of the screen for $1,000 \mathrm{msec}$, followed by a blank field for $500 \mathrm{msec}$. The cue was then displayed, centered on the screen, until the subject responded. Response time was measured from cue on-screen to keypress. After an intertrial interval of $2,000 \mathrm{msec}$, the next trial followed automatically. The subject was instructed to "bring to mind a memory of a personal experience which took place over the course of minutes or hours." Memories relating to longer time periods were not acceptable. It was emphasized that the subject was not to select particular memories, and that as soon as a memory came to mind the subject should press the response key with the dominant hand. Further instructions, relating to the prime and to failure to recall a memory, were similar to those employed in Experiment 1.

It was explained to subjects that detailed accounts of their memories would not be required but that they would be asked some general questions about their memories at the close of the experiment. This part of the experimental sequence took about $10 \mathrm{~min}$. All subjects then took part in an informal postexperimental interview.

\section{Results and Discussion}

For the purpose of analysis, the two different types of prime-cue pairs, personal and semantic, were treated as separate subexperiments. We reasoned that the personal prime-cue pairs differed from the semantic prime-cue pairs in a variety of ways that made them not directly comparable. For instance, the semantic prime-cue pairs were repeated across subjects, whereas the personal prime-cue pairs were unique to each subject. Furthermore, the semantic cues were drawn from categories with a limited number of exemplars and with only a small number of highly typical exemplars. The personal cues were drawn from lifetime periods, which, presumably, contained a vast number of personal experiences. We decided, however, to perform comparisons between the two sets of retrieval times in cases where the analyses indicated that this would be appropriate.

Prior to analysis, the distributions of the retrieval times were examined and those in excess of $30,000 \mathrm{msec}$ were treated as errors and replaced by the subject's mean. Only four retrieval times were replaced, and there were no other errors. The distribution of retrieval times to semantic cues was highly skewed (30.06) and leptokurtic (45.09). The distribution of retrieval times to personal history cues was also found to be highly skewed (12.35) and leptokurtic (22.30).

Retrieval times to semantic cues were entered into a $2 \times 10$ within-subjects analysis of variance. The first factor was prime versus no-prime, and the second factor was cues. Cues were treated as a random factor, and $\min F^{\prime}$ ratios were calculated. No significant effect of priming was observed $\left[\min F^{\prime}(1,25)=0.089\right]$. Mean retrieval times for prime and no-prime conditions were $3,660 \mathrm{msec}$ and 3,542 msec, respectively. Thus, as in Experiment 1, semantic primes did not facilitate memory retrieval. 
An identical analysis of variance was conducted for retrieval times to personal cues. A highly significant effect of priming was observed $\left[\min F^{\prime}(1,44)=14.9\right.$, $p<.01]$. Mean retrieval times for the prime and noprime conditions were $1,841 \mathrm{msec}$ and $2,742 \mathrm{msec}$, respectively. Thus memories were retrieved $899 \mathrm{msec}$ faster to primed personal cues than to unprimed personal cues.

In order to examine the interaction of priming with semantic and personal cues, we first decided to compare the variance within the two sets of data. For each subject, a priming score was calculated by subtracting the mean retrieval time collapsed across items in the prime condition from the mean retrieval time collapsed across items in the no-prime condition. The mean square error (MSe) for each set of priming scores was then calculated. The MSe was $1,887,012 \mathrm{msec}$ for prime scores in the semantic cue condition and $749,386 \mathrm{msec}$ for prime scores in the personal cue condition. The two sets of prime scores differed significantly in their variability $[F(27,27)=$ $2.518, p<.05]$ and were not compared further.

The main finding, then, was that autobiographical memory retrieval can be primed when the prime and cue refer directly to a subject's personal history. Prior presentation of a lifetime period (e.g., "sixth form") facilitated recall of a specific autobiographical memory to a personal history cue (e.g., "holiday in Italy"), compared to the prior presentation of a neutral prime. This finding suggests that the representation of specific autobiographical memories may be in terms of more general personal knowledge, such as lifetime periods and extended events (as named by personal history cues).

One implication of this finding is that autobiographical memories may be organized in terms of time periods. The lifetime periods listed by the subjects were not, however, mutually exclusive, and all subjects named at least some lifetime periods that referred to overlapping time periods. For example, one subject listed the lifetime periods "when at university" and "when going out with Julie," which overlapped, although not fully. This suggests that lifetime periods, although they refer to time periods with marked beginnings and endings, are distinguished by general features of that time (e.g., workplace, partner). These general features may be employed to segregate memories into distinct groups of events. Thus the date of occurrence of an event, or the time period over which a similar set of events took place, is probably not a critical feature in distinguishing sets of memories.

It is suggested, then, that the lifetime period primes activated sets of general memories of extended events. These general memories delineated sets of specific autobiographical memories. Retrieval to primed personal history cues was fastest because of the spread of activation from lifetime period primes to related general events. This is discussed further below.

One shortcoming of the present experiment was that subjects were exposed twice to the personal history cues but only once to the semantic cues. Recall that 4 to 5 months prior to the priming phase of the experiment, the subjects had listed lifetime periods and personal history cues, from which a random sample of personal history cues was later selected. When presented with personal history cues in the priming experiment, then, the subjects may have been cued back to some recollection of completing the personal memory questionnaire. If, in this earlier phase of the experiment, the subjects had recalled specific autobiographical memories (although they were not instructed to do so), these may have been stored in an episodic record of personal memory questionnaire completion. Thus, personal information may have activated some subset of the memory of completing the questionnaire, rather than autobiographical memory as such. In Experiment 3, we attempted to obviate this potential confound.

\section{EXPERIMENT 3}

In order to examine the possible confound of two exposures to personal history cues, it was decided to adopt the following procedure: In addition to completing the personal memory questionnaire employed in Experiment 2, the subjects would be required to recall specific personal memories to self-generated category names and exemplars. As the personal memory questionnaire did not explicitly require the retrieval of specific memories, this procedure should positively bias priming effects for memory retrieval to semantic cues. If an episodic memory of questionnaire completion were retrieved in response to primes and cues, then, with the present procedure this memory would be more likely to contain specific autobiographical memories associated with semantic primes and cues. It would be less likely, however, to contain specific autobiographical memories associated with lifetime period and personal history primes and cues.

In order to make further comparisons between different types of cues, it was decided to employ the cues used by Reiser et al. (1985). These cues consisted of activities (e.g., "going to the cinema") and general actions (e.g., "finding a seat"). Reiser et al. found that retrieval times were shorter when cues were presented in the order activity/general action than in the order general action/ activity; thus, it seemed reasonable to assume that activities would prime sets of memories containing related general actions.

These cues are comparable to the lifetime period and personal history cues in a number of respects. First, lifetime periods, like activities, contain information about relatively proscribed contexts (e.g., "going to university"). Second, personal history cues, like general actions, specify actions that may have been undertaken in a number of (lifetime) contexts: for example, a person may have been to parties while at university and during other lifetime periods. However, personal history and general action cues differ in terms of the specificity of their per- 
sonal references. Presumably, both should produce priming effects, although the personal primes and cues may produce significantly faster retrieval times overall.

A further aspect of the experiment concerned the type of memory recalled. Rehearsal and importance have been found to be associated with highly vivid memories (Conway \& Bekerian, 1986; Rubin \& Kozin, 1984). Clearly, it is possible that different types of cues are more effective in the retrieval of vivid memories. One possibility is that personal history cues may give rise to the recall of more specific, significant, and well-rehearsed autobiographical memories. Therefore, ratings of the frequency of rehearsal of the memories, their personal importance, pleasantness, and degree of specificity were collected.

\section{Method}

Subjects. Twenty subjects, 12 male and 8 female, were recruited from the subject panel of the Applied Psychology Unit. This group of subjects completed the personal memory questionnaire. Three subjects were discarded due to incorrect and incomplete responses. Six months later, the remaining 17 subjects were asked to take part in a primed autobiographical memory retrieval experiment. One subject, however, could not be contacted. Thus, 16 subjects, 9 male and 7 female, with ages ranging from 27 to 38 years, took part in both phases of the experiment. They were paid for their participation.

Design. The design was the same as that employed in Experiment 2 , with the following exceptions: The subjects retrieved memories to four different sets of stimuli. These were old semantic cues (i.e., cues to which subjects had previously been exposed), new semantic cues, personal history cues, and general action cues. Half the cues in each set were primed with the word "ready," and the remaining cues were primed as follows: old semantic cues were primed with self-generated semantic category names, new semantic cues were primed with experimenter-selected semantic category names, personal history cues were primed with lifetime period names, and general action cues were primed with activities (see below for details of stimuli selection). There were 16 cues in each group. Thus there were three within-subjects factors: groups, primes, and items (cues).

There were five dependent measures: autobiographical memory retrieval time, ratings of personal importance of recalled events, ratings of how frequently a memory had been rehearsed, ratings of how pleasant a recalled event had been, and ratings of how specific a memory was.

Stimuli selection. In the first phase of the experiment, the subjects completed the personal memory questionnaire employed in Experiment 2. Lifetime period primes and personal history cues were selected in the same way as in Experiment 2. Also in this phase of the experiment, the subjects generated categories and exemplars to which they retrieved personal memories.

For this part of the experiment, the following procedure was adopted. Twenty semantic categories naming common objects or activities were selected from Battig and Montague (1969). From this set, 12 categories were randomly selected and 3 frequently named exemplars were selected from each category. Each group of three exemplars was read aloud to the subjects, who were instructed to write down a word or short phrase naming the category to which the exemplars belonged. The subjects were provided with a 12-page booklet. After writing each category name on a separate page, the subjects turned back to the first page of the booklet and wrote down five exemplars for each category. They were instructed not to name the exemplars they had just heard and to try to name each exemplar by a word or short phrase. They were further in- structed to name typical exemplars or exemplars "which most people would agree are a member of the category." After completing this task, the subjects turned to the first page of the booklet and recalled a specific autobiographical memory for each exemplar. They were instructed to make a short note about each memory next to the exemplar, so that reading the note at a later date would remind them of the memory they had recalled. This part of the experiment lasted about $30 \mathrm{~min}$.

All subjects provided category names similar to those in Battig and Montague (1969). Self-generated exemplars differed across subjects, but in general were clearly category members. Old semantic stimuli were selected in the following way: Exemplars that were named by the experimenter and repeated by the subject (less than $2 \%$ of all exemplars) were eliminated from the selection procedure. For each subject, eight category names were randomly selected to act as primes. Two exemplars from each category were randomly selected to act as memory cues.

The eight category names not employed as old semantic primes were employed as new semantic primes. From each category, 2 exemplars were randomly selected from the 10 most frequently named exemplars listed in Battig and Montague (1969) to be used as the new semantic memory cues.

For the activity primes, we selected eight common activities from the activity and general action stimuli of Reiser et al. (1985). Eight associated general actions that specified successful actions (see Reiser et al., 1985, p. 133) were selected to act as general action memory cues. Another eight associated general actions were generated; these were similar to those taken from Reiser et al., but specified different actions. General actions taken from Reiser et al. and generated for this experiment were randomly allocated to the primed and unprimed conditions for each subject.

Thus there were 16 memory cues and eight primes in each of the four sets of stimuli. The procedure for pairing primes and cues was similar to that employed in Experiment 2. Each subject received a different random order of presentation of the 64 memory cues. Other aspects of the experiment were the same as in Experiment 2.

Procedure. The procedure was the same as that employed in Experiment 1 , with the following changes. Six to 7 months after participating in the first session, individual subjects took part in the autobiographical memory retrieval study. In this phase of the experiment the subjects were tested by a different experimenter and were unaware (at least initially) that the experiment featured personal information.

After completing the primed retrieval part of the experiment, the subjects were provided with a booklet and the following instructions:

In this part of the experiment you are asked to provide various ratings of your memories, and, in order to do this, you must read the descriptions you made of your memory and then rate that memory in the following way:

(A) You are asked to judge how personally important the event was which you recalled. Rate the event as a 1 if it was a highly significant event in your life; rate the event a 2 if it was of moderate signifiance; and rate the event a 3 if it was of little or no personal significance.

(B) Next judge how frequently you have thought about and/or talked about that event. Rate the event as a 1 if you have thought/talked about that event fairly frequently. Rate the event a 2 if you have thought/talked about that event occasionally. Rate the event a 3 if you have rarely thought/talked about that event.

(C) Then judge how pleasant or unpleasant that event was. Rate the event a 1 if it was a fairly pleasant or happy occasion. Rate the event a 2 if it was not particularly pleasant or unpleasant. Rate the event a 3 if it was a fairly unpleasant or unhappy occasion.

(D) Finally you are asked to judge how specific your memory was. Rate the memory 1 if it was fairly detailed and specific. Rate the memory as a 2 if it was moderately detailed. Rate the memory 3 if it contained few details and was rather hazy. 
The experimenter established that the subjects understood each of the rating scales. The subjects then turned to their memory notes and rated each memory on each of the four scales.

At the end of the experiment the subjects were debriefed and asked if they had been surprised at the inclusion of personal information and if they knew the source of that information. They were also asked if they had remembered the first phase of the experiment during the course of recalling memories in the second phase.

Finally, the subjects were paid and collected their memory notes, which they took with them.

\section{Results and Discussion}

The results are reported in three sections. The first section reports an analysis of retrieval times, the second an analysis of the memory ratings, and the third a multiple regression of retrieval times and ratings. Omissions were less than 3\% and these were replaced by the subject's mean in that condition. This was done for both retrieval times and ratings. A summary of mean retrieval times is provided in Table 1.

Retrieval times. As the sets of memory cues-personal history, general action, old semantic, and new semanticcontained different cues and primes, it was decided to analyze each group separately. Identical within-subjects analyses of variance were conducted for each of the four sets of retrieval times, in which subjects and items (cues) were treated as random factors and prime was treated as fixed. Items were nested within prime. Distributions of retrieval times within cue groups were also examined.

Retrieval times to personal history cues were skewed, 8.34, with a kurtosis of 15.12. A significant effect of prime was observed $\left[\min F^{\prime}(1,28)=8.85, p<.006\right]$. Retrieval times to general action cues were skewed, 6.12 , with a kurtosis of 11.89. No significant effect of prime was found. Retrieval times to old semantic cues were skewed, 6.29, with a kurtosis of 12.66. No significant effect of prime was found. Retrieval times to new semantic cues were skewed, 6.34, with a kurtosis of 1.50. No significant effect of prime was found.

Thus, only lifetime periods were found to reliably prime retrieval of autobiographical memories cued by general events related to the lifetime periods. Semantic category primes and cues, which had been seen before and which had been used to elicit autobiographical memories, did not prime memory retrieval. It can be concluded, then, that the priming effect to personal history cues was not a product of prior exposure to the stimuli.

Unexpectedly, however, there was no significant effect of activity primes on retrieval to general action cues, and this result contrasts with those of Reiser et al. (1985).

Table 1

Mean Retrieval Times (in Milliseconds)

\begin{tabular}{lcccc}
\hline & \multicolumn{4}{c}{ Cue Type } \\
\cline { 2 - 5 } Condition & PH & AC & OS & NS \\
\hline Prime & 1921 & 3286 & 2873 & 2977 \\
No-Prime & 2521 & 3185 & 2581 & 2980 \\
\hline
\end{tabular}

Note- $\mathrm{PH}=$ personal history $; \mathrm{AC}=$ general action; $\mathrm{OS}=$ old seman tic; NS = new semantic
There were, however, a number of differences between the present experiment and that of Reiser et al. Most importantly, Reiser et al. showed activity cues (e.g., "going to the cinema") for $5 \mathrm{sec}$ prior to presentation of the general action cues (e.g., "finding a seat"), and subjects retrieved a memory that corresponded to the scene specified by both cues. In the present experiment, subjects viewed the prime (activity) for $1.5 \mathrm{sec}$ and then retrieved a memory to a related general action cue. Thus, in the present experiment, the context provided by an activity prime did not necessarily have to be employed in the process of memory retrieval, and this may have contributed to our failure to find priming effects. This suggestion, however, is somewhat tenuous, as subjects in the personal history condition also did not have to use the lifetime period prime in retrieval, yet a significant priming effect was observed.

A more plausible explanation relates to the differences in exposure time of primes. The 5-sec exposure of primes in Reiser et al.'s (1985) study perhaps allowed subjects to construct a retrieval plan on the basis of the contextual information contained in the prime. For instance, in response to the prime "going to the cinema," a subject may have had sufficient time to retrieve a lifetime period during which he/she frequently went to the cinema (e.g., "when at university"). The lifetime period would then facilitate access to personalized general events and hence to specific autobiographical memories containing records of general actions (e.g. " "finding a seat"). The control condition in Reiser et al.'s study involved priming with a general action, and this may have given rise to longer retrieval times because general actions do not map onto any single lifetime periods. In contrast, the short exposure time to activity primes employed in the present experiment may not have been long enough to allow the retrieval of an appropriate lifetime period, hence the longer and more variable retrieval times and the failure of the prime to facilitate retrieval.

This argument suggests that varying prime exposures may be one way to determine the effectiveness of different cues in influencing autobiographical memory retrieval. Abstract, semantic-like primes that specify contexts may facilitate memory retrieval, but only if sufficient time is allowed for the subject to utilize that information. Primes that specify time periods relating to a person's life appear to require less time to facilitate memory retrieval. This suggests that one way in which autobiographical memory may be organized is in the form of a hierarchically structured personal history. This point is discussed further below.

Memory ratings. Four identical analyses of variance were conducted on each of the rating scales separately. Subjects and items were treated as random effects, and groups (personal history, general action, old semantic, new semantic) and prime were treated as fixed effects. Items were nested within groups and prime. Mean ratings are shown in Table 2. 
Table 2

Mean Memory Ratings

\begin{tabular}{lcccc}
\hline & \multicolumn{4}{c}{ Cue Type } \\
\cline { 2 - 5 } Rating Scale & PH & AC & OS & NS \\
\hline Importance & 1.57 & 1.86 & 2.05 & 2.12 \\
Rehearsal & 1.95 & 2.36 & 2.37 & 2.28 \\
Pleasantness & 1.86 & 2.03 & 1.89 & 1.92 \\
Specificity & 1.41 & 1.47 & 1.68 & 1.69
\end{tabular}

Note-PH = personal history; $\mathbf{A C}=$ general action $;$ OS = old semantic; NS = new semantic. Ratings underlined by a common line do not differ significantly from each other but do differ significantly from all other ratings. All ratings range from 1 to 3 (see text for details).

Ratings of personal importance: The only significant effect was that of groups $\left[\min F^{\prime}(3,101)=9.98\right.$, $p<.001]$. In order to examine this further, NewmanKeuls tests (Winer, 1971, p. 196) were conducted across the subjects analysis and, separately, across the items analysis. Only effects that were significant on both analyses are reported here. It was found that ratings of personal importance of memories retrieved to personal history cues were significantly different from ratings of all other memories. Ratings of personal importance of memories retrieved to general action, old semantic, and new semantic cues did not differ significantly (see Table 2).

Ratings of frequency of rehearsal: The only significant effect was that of groups $\left[\min F^{\prime}(3,100)=12.15\right.$, $p<.001$ ]. This effect was examined further by NewmanKeuls analyses identical to those reported for ratings of personal importance. It was found that ratings of rehearsal of memories retrieved to personal history cues differed significantly from ratings of all other memories and that ratings of memories retrieved to general action, old semantic, and new semantic cues did not differ significantly.

Ratings of pleasantness: No significant effects were observed.

Ratings of specificity: The only significant effect was that of groups $\left[\min F^{\prime}(3,83)=5.35, p<.002\right]$. Newman-Keuls analyses identical to those previously conducted showed that ratings of specificity of memories retrieved to personal history and general action cues were significantly different from ratings of memories retrieved to old semantic and new semantic cues. No other significant effects were observed in this analysis.

Memories to personal history cues, then, gave rise to recall of events that were rated as being more personally important than events recalled to other cues, as being more frequently thought about than memories recalled to other cues, and as being more specific than memories recalled to old semantic and new semantic cues. This suggests that personal history cues were providing fairly direct access to autobiographical memories. Memories retrieved to general action cues were rated as being as specific as memories retrieved to personal history cues, perhaps indicating that these cues were effective in inducing access to autobiographical memories but not to memories of events of any significance. Also, as Reiser et al. (1985) found, the rated pleasantness/unpleasantness of the recalled events was unrelated to cue type, primes, and retrieval time.

Retrieval times and memory ratings. In order to examine relationships between retrieval times and memory ratings, it was decided to conduct a two-phase multiple regression with retrieval time as the dependent variable and subjects, prime, and the four different ratings as predictor variables. Because of differences in variance among cue groups, regressions were performed for each cue group separately. In the first phase of the regression, only subjects and prime were entered as factors. In the second phase, the four memory ratings were also included in the analysis. Unless otherwise stated, all percentages of variance reported below are significant $(p<.05)$.

Memories retrieved to personal history cues: Subjects and prime were found to account for $47.6 \%$ of the variance in retrieval times, with individual subjects accounting for the largest proportion of this variance. When memory ratings were included in the analysis, the variance accounted for rose to $48.8 \%$. Thus, the memory ratings accounted for $1.2 \%$ of the retrieval time variance. Different memory ratings accounted for significant amounts of this variance; however, because the overall amount of variance accounted for by the ratings was so small, this aspect of the analysis is not reported further.

Memories retrieved to general action cues: Subjects and prime were found to account for $41.7 \%$ of the retrieval time variance, with individual subjects accounting for the largest proportion of this variance. Memory ratings were found to account for an additional $1.7 \%$ of the variance, making the total variance accounted for $43.4 \%$.

Memories retrieved to old semantic cues: Subjects and prime accounted for $16.9 \%$ of the retrieval time variance, with individual subjects accounting for the largest proportion of this variance. Memory ratings accounted for an additional $2.9 \%$ of the variance, making the total variance accounted for $19.8 \%$.

Memories retrieved to new semantic cues: Subjects and prime accounted for $30.9 \%$ of the retrieval time variance, with individual subjects accounting for the largest proportion of this variance. Memory ratings accounted for an additional $1.1 \%$ of the variance, and this was not significant $[F(4,235)=1.94]$. Thus, the total variance accounted for was $32.0 \%$.

In general, the memory ratings accounted for only a small percentage of the variance in retrieval times, as did the prime manipulation; however, as primes had a significant effect in only one group (personal history), this is hardly surprising. Across all of the above regression analyses, subject variance was found to be the best predictor of retrieval time. Nevertheless, combinations of all of these factors failed to account for more than $50 \%$ of the retrieval time variance and less than $30 \%$ of the variance for retrieval times to semantic cues. These findings suggest that the process of autobiographical memory access may be subject to many factors and that, even with 
primes and cues that relate to a person's life, other factors may play an important role in determining retrieval time. Williams and Hollan (1981) characterized autobiographical memory retrieval as a problem-solving process and provided some evidence that variability in retrieval accuracy may relate to fluctuations in performance at many different points in the retrieval process. Presumably, such fluctuations occur both within and between subjects. If such is the case, then it is hardly surprising that much of the variability in retrieval time in the present experiment could not be accounted for. This suggests that retrieval time alone may not be a sensitive measure of autobiographical memory retrieval and that other measures must also be employed.

Finally, it should be noted that in Experiment 3, as in Experiment 2, there was quite a considerable overlap between different subjects' lifetime periods and personal history cues. Over $70 \%$ of the lifetime period primes were common to all subjects ("preschool years," "primary school," "high school," "college," "first job," "'married life," "after children were born," etc.). Furthermore, the lifetime period primes that were not the same, word for word, across subjects nevertheless named similar types of events (serious illnesses of various sorts, different periods of employment, times spent with different partners, etc.). These lifetime periods were, then, general to subjects and contained little idiosyncratic information. As such, they may be comparable to abstract semantic knowledge. In contrast, personal history cues referred to distinctive general periods that were primarily unique in the context of a lifetime period (holidays, terms at college/university, periods of progress/failure at work, different phases of married life, etc.). Although the information contained in these cues was specific to individuals, the types of events were similar across subjects. Thus, it seems that idiosyncratic information, which differs from that of others in detail but not in type, about one's personal history is further represented under higher level information (lifetime periods) that is common in both detail and type to that of other people.

Finally, in the informal postexperimental interview, all subjects claimed to have recognized, and been surprised by, the personal history cues. No subject recalled when he/she had divulged this information. All subjects assumed that the cues had been derived from information collected across the many studies in which they had previously participated. It seems reasonable to conclude, then, that subjects did not consciously recall the first phase of the experiment during the priming study.

\section{GENERAL DISCUSSION}

In Experiment 1 it was found that semantic category primes did not facilitate the recall of autobiographical memories cued by category exemplars, and that retrieval times were highly variable. The category Emotions, however, gave rise to significantly longer retrieval times than did the categories Sports and Furniture. In Experi- ment 2 it was found that autobiographical memory retrieval could be facilitated when the primes referred to periods from a subject's life and the cues referred to general events associated with those periods. Retrieval times in this experiment were faster than those to semantic primes and cues, and somewhat less variable. Experiment 3 replicated the priming effects observed in Experiment 2, and found that activity primes and general action cues, taken from Reiser et al. (1985), did not facilitate autobiographical memory retrieval.

In Experiment 3 it was also found that memories recalled to personal history cues differed in other ways from memories recalled to other cues. Memories recalled to personal history cues were rated as being highly specific, frequently rehearsed, and personally important. Memories recalled to other types of cues were rated as being less specific (although this was not the case for memories recalled to general action cues), less frequently rehearsed, and of less personal importance. Memory ratings, however, were found to account for little of the retrieval time variance; similarly, the experimental manipulations accounted for less than half of this variance. Together, the results of the three experiments suggest that one principal way in which autobiographical memory may be organized is in terms of an abstracted personal history.

\section{AUTOBIOGRAPHICAL MEMORY ORGANIZATION}

The principal findings in Experiments 2 and 3 were that the recall of autobiographical memories was fastest when cued by personal history cues and that retrieval time was facilitated when these cues were primed by a lifetime period. No priming effects were found for retrieval times to the cues employed by Reiser et al. (1985). However, the personal information employed in Experiments 2 and 3 was similar to Reiser et al.'s stimuli inasmuch as activities and contexts were specified, although these did not appear to be related in any systematic way. In particular, it was not clear whether lifetime periods specified activities and/or contexts; for example, the lifetime period "primary school" (named by all subjects) could refer to an activity ("going" to primary school), to the context of primary school, or both. Thus, the findings indicate that activities and contexts are closely involved in autobiographical memory retrieval but that this type of information is most effective in the metacontext of a specific person's life. Organization within such a metacontext may not rely on systematic relations between actions, activities, and contexts.

This, however, does not mean that the models of autobiographical memory proposed by Reiser et al. (1985) and Kolodner (1983) cannot accommodate the present findings. For instance, these findings might be conceptualized in terms of what Schank (1982) called memory organization packets (MOPs). MOPs refer to memory structures that "group together actions with a shared goal, that occurred at the same time" (Schank, 1982, p. 95). 
Kolodner (1983) developed the concept of a MOP to encompass knowledge structures that "organize similar episodes according to their differences and keep track of their similarities" (p. 249). Because these knowledge structures organize memory for events, they are referred to as episodic memory organization packets (E-MOPs). E-MOPs, then, have fairly direct associations with specific groups of memories. The general events employed as personal history cues in Experiments 2 and 3 may reflect E-MOP knowledge structures. For example, general events such as "holiday in Italy" or "playing squash with Jenny" may refer to groups of memories that share common actions, locations, and actors, and which occurred within a proscribed period of time. Such E-MOPs may share certain features (e.g., time period, actors) but differ on others (e.g., location, actions).

Although the concept of an E-MOP corresponds to the concept of personal history cues, it is difficult to see how this concept can be employed to account for lifetime periods. In Schank's (1982) and Kolodner's (1983) models, MOPs and E-MOPs are characterized as existing at different levels of specificity. According to these models, lifetime periods are highly abstracted representations with the same function and structure as MOPs and/or E-MOPs. This characterization, however, fails to capture important ways in which lifetime periods differ from E-MOPs. For example, lifetime periods were found to be similar across subjects in both detail and type, whereas E-MOPs (general events), although showing some similarity in type, differed in their specific details from subject to subject. Furthermore, lifetime periods appear to be directly associated not with specific autobiographical memories, but rather with E-MOPs. An additional possibility is that the information contained in lifetime periods differs from E-MOP information. In Kolodner's model, E-MOPs contain information about actors, actions, locations, and activities. Lifetime periods are unlikely to contain such specific information, but are usually more abstract, listing extended periods with few details. The details of lifetime periods may be specified by the E-MOPs they index.

Related to this, Conway, Bekerian, and Cohen (1987) found that when subjects were asked to list significant life events that might be experienced by the average British citizen, the most frequently named events closely corresponded to the lifetime periods observed in the present study (see also Bekerian \& Conway, 1987b). Taken together, these findings suggest that lifetime periods may constitute a category that, in an abstract way, summarizes general aspects of an individual's autobiography and, because of similarities across subjects, integrates different personal histories (at least within a culture).

This category of lifetime periods might be conceptualized as an autobiographical memory organization packet (A-MOP), a single knowledge structure that contains abstract summaries of a vast number of memories, such as "school days," "married life," "when children were lit- tle," "first job," and so on. Presumably, this knowledge structure is based partly upon actual experiences and partly upon socialization experiences. That is to say, within a culture, it is expected that people will experience certain lifetime periods and this may predispose an individual to represent sets of experiences in terms of culturally specified norms.

The present findings suggest that members of the AMOP (e.g., "school days") index related E-MOPs (e.g., "math class"), which in turn index specific autobiographical memories. Thus, the prior presentation of a lifetime period may have activated a related set of general events which in turn activated related sets of specific autobiographical memories. Retrieval of a specific memory to a subsequently presented general event (E-MOP) would, then, have been facilitated by the earlier activation effect of the A-MOP prime, hence, retrieval times were fastest in this condition. Faster retrieval times overall to personally relevant cues indicate that these cues were specific to each individual's knowledge structures.

One implication of the above proposal is that autobiographical memories are represented in memory in a particular way (i.e., in some form of hierarchical system). There is, however, an alternative interpretation of the present findings that makes no strong commitment to representation. It could be argued that the personal primes and cues were most effective in the construction of retrieval plans and that these plans were used to search a relatively unstructured set of memories. Longer retrieval times to other cues may have reflected the lengthier implementation of a similar form of retrieval. It is not apparent how these two positions might be experimentally distinguished, especially as they may entail similar predictions. Prestored versus computed models of memory access have been discussed before (see Smith \& Medin, 1981). Furthermore, Hollan (1975) pointed out that the two positions differ mainly in terms of theoretical preferences, rather than in terms of predictions.

It is not clear how the personal primes and cues employed in the present study could have selectively facilitated the implementation of retrieval plans/strategies if memories were not already represented in such a way as to make those cues highly salient to the recall process. Thus, although the computed model of memory access remains a possibility, the preference here is for an explanation that emphasizes representation. Of course, some form of plan or strategy may be constructed in the process of accessing hierarchically structured personal knowledge.

For example, when presented with a cue that does not correspond to the way in which memories are represented, a subject may have to elaborate the cue in order to initiate retrieval. This type of explanation may account for the failure to find priming effects in Experiment 3. It may also suggest why, in Experiment 1, no priming effects were observed and retrieval times generally were highly variable, although retrieval times to cues naming Emotions were significantly longer than those to cues naming items of Furniture and Sports. Because no personal in- 
formation was presented in this experiment, it may have been the case that part of the retrieval process entailed the generation/search of lifetime periods judged likely to index an appropriate specific memory. Possibly, the generation of A-MOP information and/or the indexing of related E-MOPs was more difficult for emotion cues than for activity or object cues, hence the longer retrieval times to emotion cues. As Robinson (1976) implied, this may be because emotion cues refer to many contexts. If such were the case, the specificity of personal contextual information would be reduced, which would have the effect of slowing retrieval processes employing such information.

Clearly, further investigations of this category effect are required. It may be that other factors effect the retrieval of autobiographical memories of emotional experiences. For instance, Conway and Bekerian (in press) found that recall of emotional experiences gave rise to marked mood shifts; it may be that the category effect observed in Experiment 1 was, in part, a product of a mood incongruence effect (Bower, 1981).

The structure of autobiographical memory proposed above does not, however, mean that autobiographical memories are always accessed through a hierarchy of personal information. The incidental recall of autobiographical memories in everyday life may be initiated by contextual cues that access specific memories directly. Cues such as smells may operate in this way, and there are, of course, many other examples. Nevertheless, active searches for specific autobiographical memories appear to involve the use of an A-MOP and associated E-MOPs.

Consider the following two protocols, collected from 2 subjects who were asked to "think aloud" while they responded to the instruction "Tell me a specific detailed experience from your own life which occurred when you were 18 years old":

I was in my first year at university and I remember going with Gerry for a drink in the Union bar.... It was just before the Christmas holidays.... I remember how dark the bar was. We spent most of our time kissing. (a 33-yearold male subject)

I was in my final year at school. We had just moved house and the "new math" had been introduced at school. I remember a 2-hour math class in which we learned about gradients. (a 27-year-old female subject)

Both protocols contain lifetime periods that might be represented in an A-MOP ("when at university," "when at school"), E-MOPs in the form of related general periods ("end of first term," "learning math"), and distinctive details of specific events ("kissing," "gradients"). Other general periods are also mentioned ("Christmas holidays," "moving house"), although these appear to be less directly related to the lifetime periods.

In summary, the present findings indicate that general personal information, in the form of a category of lifetime periods (an A-MOP) and related general events (EMOPs), provides faster access to specific autobiographi- cal memories than do other types of information. General personal information also led to the recall of more specific memories, which had been frequently rehearsed and which were judged as being memories of personally significant events. Autobiographical memory may, then, be organized in a hierarchy of personal information. This hierarchy ranges from abstract to specific knowledge, and more abstract levels of the hierarchy index more specific levels: lifetime periods (A-MOP) index general events (E-MOPs), which in turn index specific autobiographical memories. More abstract personal knowledge may be common to individuals who may share similar, if not identical, lifetime periods. Some commonality between EMOPs was also observed. More specific personal knowledge, in the form of autobiographical memories, is idiosyncratic to individuals.

\section{REFERENCES}

ANDERSON, J. R. (1983). The architecture of cognition. Hillsdale, NJ: Erlbaum.

Battig, W. F., \& Montague, W. E. (1969). Category norms for verbal items in 56 categories: A replication and extension of the Connecticut category norms. Journal of Experimental Psychology Monograph, 80(3, Pt. 2)

BEKERIAN, D. A., \& Conway, M. A. (1987a). Autobiographical memory content. In preparation.

Bekerian, D. A., \& Conway, M. A. (1987b). Everyday contexts. In G. Davies \& D. Thomson (Eds.), Memory in context: Context in memory. London: Wiley.

Bower, G. H. (1981). Mood and memory. American Psychologist, 36, 129-148

Clark, H. H. (1973). The language-as-fixed-effect fallacy: A critique of language statistics in psychological research. Journal of Verbal Learning \& Verbal Behavior, 12, 335-359.

Coluins, A. M., LofTus, E. F. (1975). A spreading activation theory of semantic processing. Psychological Review, 82, 407-428.

Conway, M. A., Bekerian, D. A. (1986). Vivid memories: Encoding or recoding? Manuscript submitted for publication.

Conway, M. A., Bekerian, D. A. (in press). The role of situations in knowledge of emotions. Cognition \& Emotion.

Conway, M. A., Bekerian, D. A., Cohen, G. (1987). Significant life-events and autobiographical memory. Manuscript in preparation.

dE Groot, A. M. B. (1983). The range of automatic spreading activation in word priming. Joumal of Verbal Leaming \& Verbal Behavior, 22, 417-436.

Fehr, B., \& Russell, J. A. (1984). Concept of emotion viewed from a prototype perspective. Joumal of Experimental Psychology: General, $113,464-486$

Fehr, B., Russell, J. A., \& WRD, L. (1982). Prototypicality of emotions: A reaction time study. Bulletin of the Psychonomic Sociery, 20, 253-254

GALTON, F. (1883). Inquiries into human faculty and its development (1st ed.). London: MacMillan.

Hampton, J. A., Gardiner, M. M. (1983). Measures of internal category structure: A correlational analysis of normative data. British Journal of Psychology, 74, 491-516.

Hollan, J. D. (1975). Features and semantic memory: Set-theoretic or network models? Psychological Review, 82, 154-155.

KfPPEL, G. (1973). Design and analysis: A researcher's handbook. Englewood Cliffs, NJ: Prentice-Hall.

KoLoDNer, J. A. (1983). Maintaining organization in a dynamic long. term memory. Cognitive Science, 7, 243-280.

Landauer, T. K. Freedman, J. L. (1968). Information retrieval from long-term memory: Category size effect and recognition time. Journal of Verbal Learning \& Verbal Behavior, 7, 291-295. 
NoRRIs, D. (1984). A computer-based programmable tachistoscope for nonprogrammers. Behavior Research Methods, Instruments, \& Computers, 16, 25-27.

Reiser, B. J., Black, J. B., \& Abelson, R. P. (1985). Knowledge structures in the organization and retrieval of autobiographical memories. Cognitive Psychology, 17, 89-137.

Reiser, B. J., Black, J. B., Kalamarides, P. (1986). Strategic memory search processes. In D. Rubin (Ed.), Autobiographical memory. New York: Cambridge University Press.

Robinson, J. A. (1976). Sampling autobiographical memory. Cognitive Psychology, 8, 578-595.

Rosch, E. (1975). Cognitive representations of semantic categories. Journal of Experimental Psychology: General, 104, 192-233.

RubIN, D. C., \& KozIN, M. (1984). Vivid memories. Cognition, 16, 81-96.

SCHANk, R. C. (1982). Dynamic memory: A theory of reminding and learning in computers and people. New York: Cambridge University Press.

Smith, E. E., \& Medin, D. L. (1981). Categories and concepts. Cambridge, MA: Harvard University Press.

Williams, M. D., \& Hollan, J. D. (1981). The process of retrieval from very long-term memory. Cognitive Science, 5, 87-119.

WINER, B. (1971). Statistical principles in experimental design. New York: McGraw-Hill.

\section{NOTE}

1. In all of the experiments reported in this paper, two types of analysis were performed: the raw data were analyzed, and the data were $\log$ transformed. The results of these two types of analysis did not contradict each other for any of the experiments; therefore, the analysis of raw data is reported. However, it should be noted that in all cases the $\log$ transform reduced both skew and kurtosis and produced data sets that approximated normal distributions. Thus the distributions reported appear to be distorted by a small number of outliers and by the close clustering of the majority of retrieval times.
APPENDIX

Stimuli Employed as Memory Cues in Experiment 1

\begin{tabular}{|c|c|c|c|c|c|}
\hline \multicolumn{2}{|c|}{ Emotions } & \multicolumn{2}{|c|}{ Furniture } & \multicolumn{2}{|c|}{ Sports } \\
\hline Exemplar & Rating* & Exemplar & Rating* & Exemplar & Rating* \\
\hline \multicolumn{6}{|c|}{ Typical } \\
\hline anger & 1.24 & chair & 1.000 & rugby & 1.000 \\
\hline excitement & 2.15 & couch & 1.216 & badminton & 1.133 \\
\hline joy & 1.58 & wardrobe & 1.216 & golf & 1.733 \\
\hline iilt & 2.38 & cupboard & 1.6 & soccer & 1.000 \\
\hline grief & 1.2 & table & 1.0 & tennis & 1.022 \\
\hline hate & 1.51 & bed & 1.176 & swimming & 1.400 \\
\hline pleasure & 2.31 & desk & 1.5 & boxing & 1.956 \\
\hline & 1.86 & stool & 1.706 & running & 1.556 \\
\hline \multicolumn{6}{|c|}{ Atypical } \\
\hline hope & 3.79 & ashtray & 5.137 & snooker & 2.689 \\
\hline shyness & 4.10 & carpet & $\dagger$ & fishing & 3.156 \\
\hline sympathy & 3.54 & bath & $\dagger$ & hiking & 4.156 \\
\hline suspicion & 4.39 & curtains & $\dagger$ & climbing & + \\
\hline kindness & 4.03 & lamp & $\dagger$ & karate & 2.867 \\
\hline greed & 4.18 & television & $\dagger$ & surfing & 3.267 \\
\hline satisfaction & 3.80 & cooker & $\dagger$ & dancing & 5.156 \\
\hline pain & 4.58 & fridge & $\dagger$ & cycling & + \\
\hline
\end{tabular}

*1 = highly typical and 5 = highly atypical. Ratings for Emotions taken from Conway and Bekerian (in press). †Listed in Hampton and Gardiner (1983) but not given a typicality rating. All are highly atypical in Rosch (1975, Experiment 1).

(Manuscript received December 16, 1985; revision accepted for publication August 4, 1986.) 